\title{
DE LA NOCIÓN DEL CASO TRÁGICO EN LA TEORÍA DE LA ARGUMENTACIÓN JURÍDICA ESTÁNDAR Y SU PROYECCIÓN PARA EL DESARROLLO DE UNA CATEGORÍA DE ANÁLISIS EN EL ÁMBITO DE LA APLICACIÓN DE LA NORMA
}

\author{
María Andrea Figueroa Alfonzo* \\ Recibido: Abril 26 de 2016 \\ Aprobado: Junio 2 de 2016
}

\begin{abstract}
RESUMEN
El documento presenta una aproximación a la noción y los criterios señalados para el caso trágico expuesto en la Teoría de la argumentación estándar. Seguidamente se describe una sentencia constitucional colombiana que fue identificada como trágica por la Corte Constitucional. Finalmente se presenta una breve disertación que propone una hipótesis para ampliar el campo de análisis del caso trágico en el Derecho.
\end{abstract}

Palabras clave: Caso Trágico, Argumentación Jurídica, lógica, decisión judicial.

THE CONCEPT OF THE TRAGIC CASE IN THE STANDARD THEORY OF THE LEGAL ARGUMENTATION AND ITS PROJECTION FOR THE DEVELOPMENT OF A CATEGORY OF ANALYSIS IN THE AREA OF THE IMPLEMENTATION OF THE LEGAL NORM

\begin{abstract}
The article presents an approximation to the concept and criteria stipulated for the so-called tragic cases, exposed in the theory of standard argumentation. The article then describes a constitutional Colombian ruling that was identified as "tragic" by the Colombian Constitutional
\end{abstract}

* Abogada especialista en Derecho Público de la Universidad Autónoma de Bucaramanga (UNAB). 
Court. Finally, it presents a brief dissertation that proposes a hypothesis to extend the area of analysis of the tragic case in the Law.

Keywords: Tragic case, legal argumentation, logic, court decision.

\section{DA NOÇÃO DO CASO TRÁGICO NA TEORIA PADRÃO DA ARGUMENTAÇÃO JURÍDICA E SUA PROJEÇÃO PARA O DESENVOLVIMENTO DE UMA CATEGORIA DE ANÁLISE NO ÂMBITO DA APLICAÇÃO DA NORMA}

\section{RESUMO}

$\mathrm{O}$ artigo apresenta uma abordagem à noção e os critérios descritos para o caso trágico exposto na teoria padrão de argumentação. Em seguida, se descreve uma decisão constitucional colombiana que foi identificada como trágica pelo Tribunal Constitucional. Finalmente, se apresenta uma breve dissertação que propõe uma hipótese para ampliar o campo da análise do caso trágico no Direito.

Palavras-chave: caso trágico, argumentação jurídica, lógica, decisão judicial.

\section{INTRODUCCIÓN}

El documento que se presenta propone ampliar el enfoque original sobre tema del caso trágico y corresponde a un ejercicio teórico, más exactamente a las notas preliminares de una discusión llevada a cabo en el proyecto de investigación titulado "La doctrina del 'caso trágico' en la jurisprudencia de tutela de la Corte Constitucional" que fue desarrollada en el Grupo de Investigación de Hermenéutica Jurídica del Centro de investigaciones Socio-Jurídicas Laureano Gómez Serrano de la Universidad Autónoma de Bucaramanga ${ }^{1}$.

Este texto debe entenderse como una exploración crítica y alternativa sobre la idea que tradicionalmente ha sido expuesta por los principales doctrinantes que reconocen su existencia, ya que en este documento no se le da el entendimiento como una clasificación para la argumentación o un límite a la racionalidad. Por el contrario, defiende la noción del caso trágico como una categoría de análisis crítico respecto a esos elementos que inciden en la actuación del operador jurídico en el ámbito de la 
ejecución de la norma en la que se genera la tragedia.

La importancia de este ensayo radica en que participa del debate sobre el caso trágico expuesto en la Teoría de la Argumentación Jurídica Estándar del Derecho, en cuanto amplía el campo de su análisis. Desde luego, es una invitación al debate, antes que la conclusión de este tema.

El texto estructuralmente se divide en dos partes: una primera, expositiva, que describe brevemente los elementos que permiten identificar el denominado caso trágico en la teoría de la argumentación jurídica del profesor Manuel Atienza; y una segunda, analítica, donde se pretende dar los pasos iniciales para redefinir la noción de caso trágico y poder replantearlo como una categoría de análisis.

\section{SOBRE LA TEORÍA DE LAARGUMENTACIÓN JURÍDICA}

El profesor Atienza (1991) acertadamente señala que "la práctica del Derecho consiste de manera fundamental en argumentar" (p.13) y es que el Derecho obedece, en su creación y aplicación, a una variedad de factores y discursos que intentan armonizar la sociedad a través de las normas, que comprende no sólo la norma jurídica en sentido estricto, también los principios, conceptos, instrumentos de interpretación y teorías que permiten un ejercicio racional y razonable para dirimir los conflictos y cumplimiento de derechos y obligaciones descritos en la Constitución y las leyes.

En este sentido, Atienza elabora un marco teórico para justificar las razones en el Derecho denominada argumentación jurídica estándar con el fin de superar la simple adecuación de la lógica formal, propia de un Estado legalista, hacia las exigencias del Estado Constitucional y Social de Derecho, que requiere la implementación de una lógica material, pues el Derecho no puede limitar su carácter sustancial y procesal a la correcta presentación de formatos o minutas, y por el contrario debe comprender la extensión misma de la juridicidad de la jurisprudencia, de la teleología y filosofía jurídica.

\subsection{De lo razonable}

De lo anterior se desprende la importancia de hablar sobre lo razonable, que en la práctica jurídica es lo que permite establecer qué premisa es preferible o está mejor justificada que otra, por lo que este concepto se hace importante y empieza a ocupar un lugar central en algunas teorías 
de la argumentación. La noción de razonabilidad es un componente común de los que suelen llamarse conceptos jurídicos indeterminados (Atienza, 1987, p. 189), lo cual significa que tienen un carácter general aplicable a cualquier argumentación jurídica y en cierto tipo de argumentación específica, es decir es una noción variable de acuerdo con el campo de aplicación.

Siguiendo con la idea anterior, el concepto de razonabilidad es un concepto de común referencia dentro de la teoría de la argumentación jurídica. El primero en definir dicho concepto fue Recaséns Siches (Atienza, 1987, p. 90), quien consideró que la razonabilidad es un complemento de la insuficiencia de la lógica tradicional también llamada lógica racional, pues no agota la totalidad del logos de la razón y es insuficiente para tratar problemas prácticos como el de la interpretación del Derecho.

Posteriormente, Perelman intentó explicar el concepto de razonabilidad partiendo del concepto opuesto de irrazonabilidad, la cual definió como el límite negativo de cualquier argumentación que resulta inadmisible para una comunidad en un momento determinado.

Es por este motivo que, según estos autores, el límite de lo razonable lo tiene el auditorio; es decir, es la sociedad quien determina cuáles argumentos son admisibles e inadmisibles. Lo razonable, entonces, depende de una moral colectiva, de una escala de valores que determina qué es lo bueno y lo malo en una sociedad.

Razón y racionabilidad -destaca Atienza(1987) de Perelman- están unidos con ideas de verdad, coherencia y de eficacia. Lo razonable y lo irrazonable están ligados a un margen de lo admisible y lo socialmente inaceptable (por el auditorio). Sin embargo, Atienza resalta de la obra de Perelman que su teoría adolece de ambigüedad y es criticable en el sentido de que admite que dos decisiones puedan resultar considerándose como aceptables (p.191).

En el texto de 1987, Atienza enfatiza lo dicho por MacCormick en el sentido de que lo razonable se halla en "un equilibrio entre exigencias contrapuestas", y por Aaranio en que "la decisión logre aceptabilidad o consenso" (p.191). Para Atienza ambas concepciones son complementarias y le permiten elaborar un concepto de razonable para así establecer las características de esta complementariedad y sirva como criterio para entender "por qué una determinada interpretación o 
decisión es preferible a otra" (p. 191).

Aunque lo "razonable" se puede predicar de una norma jurídica o un agente jurídico, el interés de él se centra sobre el acto de "interpretar y aplicar enunciados jurídicos a casos" que terminan en decisiones jurídicas, más específicamente de los enunciados "que son el resultado de la decisión de los casos" y no de los que se utilizan para decidir los casos (Atienza, 1987, p.192). Seguidamente propone tres grupos de decisiones jurídicas:

a) Las razonables pero no estrictamente racionales.

b) Las razonables y estrictamente racionales.

c) Las no razonables (p. 192).

Las primeras son las que le interesan a Atienza, pero para su comprensión no pueden desligarse las del tercer grupo.

Una decisión es razonable en sentido estricto cuando cumple estas características:

- Es tomada en situaciones en que no sería aceptable o, no se podría, adoptar una decisión estrictamente racional.

- Logra un equilibrio entre exigencias contrapuestas, que necesariamente hay que considerar en la decisión (siendo esas exigencias principios prima facie significa que el caso bajo estudio se subsume en un amplio supuesto de hecho).

- Es aceptada por la comunidad. (p. 193)

\section{A. Racionalidad estricta}

Dice Atienza que la racionalidad es una cuestión procedimental, mientras que lo razonable se dirige hacia un resultado que debe ser argumentado y aceptable; entonces hablamos de decisiones razonables cuando no sólo siguen criterios procedimentales, sin dejar atrás que lo razonable también es racional, por eso se dice que "todo lo razonable es racional y no todo lo racional es razonable", pues es posible que una decisión se tome según criterios de estricta racionalidad, pero que resulte ser irrazonable, o sea produciendo resultados inaceptables, que son al menos dos tipos:

(i) contradicción entre los resultados que produciría la decisión y los propósitos que se persiguen por el sistema jurídico. Aquí la racionalidad estricta equivale a actuar "de una manera puramente 
ritualista".

(ii) contradicción entre la decisión y los fines o valores no contemplados en el sistema jurídico, o contradictorios con los que sí incorpora.

Destaca Atienza que identificar cuándo una decisión lleva a resultados inaceptables es más difícil en el segundo caso que en el primero.

\section{B. Equilibrio mínimo}

Resalta Atienza que la cuestión de razonabilidad no se plantea frente a los casos fáciles, sino a los difíciles. Un caso difícil se presenta si aplicando la racionalidad estricta:

i. El caso no tiene solución.

ii. Tiene una solución que resultaría inaceptable; para llegar a ella se tendría que obviar una fuente vinculante.

iii. Tiene más de una solución que son incompatible con otras. Se debe optar por la más razonable o construir una si se entiende que las que se tienen no sirven mediante criterios de razonabilidad estricta.

En estos tres casos, no es posible llegar a una decisión razonable a través de la estricta racionalidad. En ellos hay una contraposición de valores o principios que deben sopesarse entre sí hasta llegar a un equilibrio, según Atienza (1987) que no es igual a un "término medio" (p.195) pues los principios no pueden tener el mismo peso, por lo "que el equilibrio se encontraría entonces en un punto más próximo a un extremo que a otro". Este equilibrio no es permanente, tiene que construirse en cada caso para evitar que un equilibrio entre exigencias contrapuestas cause una jerarquización entre estas, de manera que unas excluyan completamente a otras.

\subsection{Campos jurídicos de la argumentación}

En la práctica del Derecho, Atienza (2005) propone tres ámbitos en los que se desarrolla la argumentación: La producción o establecimiento de normas, la aplicación de normas judiciales y la dogmática jurídica, cuya labor consiste en argumentar para la solución de casos ya sean individuales, genéricos, reales o ficticios.

\section{A. Producción de normas}

La argumentación se observa en dos fases: una prelegislativa, que se 
refiere a los problemas presentes en la cotidianidad sin elementos jurídicos, cuyos argumentos son de carácter político o moral; y una legislativa, que se encarga de problemas que pasan a consideración del Congreso, por lo que sus argumentos son de carácter técnico-jurídico (Atienza, 2005).

\section{B. Dogmática Jurídica}

La Dogmática Jurídica corresponde al conjunto de estudios del análisis jurídico del Derecho positivo vigente en un estado determinado. La argumentación de la dogmática jurídica no es muy diferente a la que hacen los órganos jurisdiccionales, porque mientras los órganos aplicadores resuelven casos individuales, la dogmática jurídica resuelve casos genéricos y tiene como función suministrar criterios para la producción y aplicación del Derecho y la sistematización de un sector del ordenamiento jurídico (Atienza, 2005).

\section{C.Aplicación de la norma}

Es la actividad que llevan a cabo todos los operadores jurídicos, su argumentación se centra en problemas concernientes a los hechos y en especial al Derecho, que son los llamados problemas de interpretación.

Cuando se plantean los problemas de interpretación se hace necesario hacer referencia a la argumentación jurídica implícita en la práctica del Derecho; por ejemplo, cuando los jueces dictan sus sentencias, cuando los abogados formulan sus pretensiones o cuando los legisladores producen sus leyes. Dentro de este contexto se distinguen tres formas de argumentación: formal, material y nuclear ${ }^{2}$, también llamada dialéctica.

La argumentación formal se refiere a aquella que se fundamenta en el silogismo jurídico. Por este se entiende una inferencia de carácter lógico en la que existe un encadenamiento de proposiciones, por lo que las conclusiones dependen del cumplimiento de reglas formales, como ocurre en el silogismo tradicional (Atienza, 1997). En otras palabras, es una tarea subsuntiva del caso a una norma legal. Este tipo de

2 La argumentación nuclear de la que aquí se hace referencia no es propuesta por Atienza, sino desarrollada teóricamente por José Guillermo CASTRO AYALA, “Otra vez sobre los casos trágicos. La previsibilidad de la decisión judicial: ¿posible o utópica? tal vez necesaria, en http://jurastudio.blogger.de/static/antville/ jurastudio/files/otra $\% 20 \mathrm{vez} \% 20$ sobre $\% 2010 \mathrm{~s} \% 20$ casos $\% 20$ tragicos.pdf, fecha de consulta 20 de febrero de 2014, p.3. 
argumentación es predominante en la solución de los casos fáciles, porque presume la existencia de una norma para su solución (Castro, s.f., p. 3).

Por otro lado se encuentra la argumentación material, aquella que se preocupa por descubrir y examinar las premisas que sirven de fundamento para llegar a la conclusión del silogismo. Para llegar a la conclusión, según Castro (s.f.), se hacen necesarias buenas razones y presentar argumentaciones adicionales a favor de la premisa que conduzcan a la posibilidad de su validez (p.2).

Por último se observa la argumentación nuclear, que autores como Castro Ayala la han definido como una argumentación dialéctica correspondiente a la mixtura entre la argumentación material y formal. Así, se encuentra que las justificaciones lógica y material que se utilizan en la resolución de casos no son excluyentes, por el contrario, pueden entenderse como complementarias, pues una justificación lógica necesariamente debe demostrarse a través de buenas razones que permite la justificación material, es decir, que hay validez, tanto en la premisa normativa como en la premisa fáctica, lo cual permite que la decisión sea aprobada. Es el nivel argumentativo en el cual el juez realiza una discusión, para imponer su criterio que por lo general corresponde a casos que no han sido previstos por el legislador, motivo por el cual se dice que existe la posibilidad de la creación de Derecho por el juez y corresponde a casos de gran complejidad como los casos trágicos. Lo que se pretende con la existencia de los grados argumentativos es garantizar una seguridad jurídica y de ahí llegar a la importante previsibilidad judicial.

\section{Clasificación de casos (fácil, difícil, trágico)}

Según Atienza y otros autores, dentro del ordenamiento jurídico se identifican los casos fáciles, difíciles y trágicos, cuya distinción depende de la teoría desde la cual se observa el caso y según la complejidad que requiere la decisión, por lo que se hace importante que el juez en el momento de dar solución a un caso pueda identificarlos, como se observará a continuación:

En primer lugar se definen los casos fáciles como aquellos casos que proveen una respuesta mediante la aplicación de un silogismo jurídico (Vázquez, 2005), por lo que el juez simplemente se encarga de obedecer una regla, que se traduce en una simple operación de 
subsunción del caso, y es en esa subsunción donde se encuentra la facilidad de estos casos, ya que no son controvertibles a los principios del sistema jurídico que dotan de sentido la institución o el sector normativo que se trate (Atienza, 1997).

Son casos en los que se aplica de forma pura y simple el Derecho, por eso en ellos no cabría la posibilidad de existir una deliberación, porque el juez simplemente se encarga de obedecer la regla para resolver dicha situación. Por lo anterior, hay quienes afirman, Como Castro (ss.f), que la solución de estos casos implica un nivel argumentativo formal o superficial, es decir, a la aplicación de una norma para resolver el caso.

En segundo lugar se encuentran los casos difíciles, que existen cuando la cuestión en litigio no puede ser determinada por los estándares jurídicos existentes (Atienza, 1997). Son casos en los que se puede encontrar más de un punto de equilibrio entre exigencias contrapuestas, motivo por el cual se exige labor interpretativa aún mayor que la simple subsunción de una norma, haciéndose inevitable la utilización de mecanismos como la ponderación (Pérez, (2007). Este resulta ser la materialización del principio de proporcionalidad a través del cual se podrá encontrar una solución justa. Atienza considera que un caso difícil existe cuando:

a) No hay consenso acerca de la resolución del caso en la comunidad de juristas.

b) No es un caso rutinario o de aplicación mecánica de la ley.

c) No es un caso fácil, y es decidible solamente sopesando disposiciones jurídicas en conflicto, mediante argumentos no deductivos.

d) El caso requiere un razonamiento basado en principios para su solución.

e) La solución del caso involucra necesariamente juicios morales. (Atienza, 1997)

Por último, la categoría de los casos trágicos, también denominados por Lariguet (2005) como "dilemas morales" (p.67) o conflictos irresolubles, se refieren a supuestos en los que se encuentran en colisión derechos fundamentales de la misma categoría, cuya solución implica el sacrificio total de uno de ellos, es decir, un elemento esencial considerado fundamental desde el punto de vista jurídico o moral (Atienza, 1997). En ellos el balance de razones no permite llegar a una situación satisfactoria, no tienen ninguna respuesta correcta, por lo que 
el juez debe decidir sobre qué camino tomar frente a un dilema; entonces, no sólo es necesaria la razón, sino también los sentimientos, las pasiones, porque para ser buen juez no es suficiente tener capacidad argumentativa y no siempre es posible hacer justicia por medio del Derecho, por eso dice Atienza (1997) que decidir sobre un caso trágico no es escoger sobre lo absolutamente bueno, sino lo mejor posible, pues supone que su solución significa que para garantizar un derecho se debe violar otro.

Atienza (1997) los ha definido como "aquellos supuestos en relación con los cuales no cabe encontrar ninguna solución jurídica que no sacrifique algún elemento esencial de un valor considerado como fundamental desde el punto de vista jurídico o moral" (p.15), puesto que: "no tienen ninguna respuesta correcta y que, por lo tanto, plantean a los jueces no el problema de cómo decidir ante una serie de alternativas (o sea, cómo ejercer su discreción), sino qué camino tomar frente a un dilema..." (p. 13).

En ese orden de ideas, Atienza propone la existencia de dos posibilidades de caso trágico que representan situaciones en las que es imposible decidir sin infringir el ordenamiento jurídico:

- Primero, una situación en la que el ordenamiento jurídico provee al menos una solución correcta de acuerdo con los valores de ese sistema pero que chocan con su moral, (lo trágico se deriva del contraste de ordenamientos distintos);

- Segundo, una situación en la que el ordenamiento jurídico no le permite alcanzar ninguna solución correcta (contradicción interna en el ordenamiento jurídico).

Para Lariguet (2004) definir los casos trágicos no es una tarea sencilla, porque su núcleo conceptual no está totalmente precisado y no puede hacerse simplemente acudiendo a su origen etimológico. Aun así identifica que un caso es genuinamente trágico cuando el conflicto no tiene resolución racional posible o cuando podría ser resuelto, pero esto implica un sacrificio o una pérdida moral. Hace entender además que si se acepta su existencia se deben aceptar también unos límites de la racionalidad, y esto ocurre cuando la razón no dice qué hacer en ciertos casos.

A partir de su postura se podría entender que el caso trágico es un límite racional, porque a diferencia de los casos difíciles, no tienen una 
solución racional posible, pues los principios en conflicto son inconmensurables, es decir, que no hay un valor que supere al otro y eso hace imposible que haya una respuesta correcta, dice que uno de los problemas con los casos trágicos es que muchas veces muestran cosas que les suceden a las personas, no cosas que estas hacen. Según Lariguet (2004) los límites de la racionalidad y sus consecuencias están lejos de ser claras tanto en el dominio general de la Filosofía práctica como en el dominio particular del Derecho (p.16).

Es así como en la postura de Lariguet se encuentra la inconmensurabilidad de los valores en los casos trágicos, elemento importante para la comprensión de la decisión que se toma sobre ellos, ya que la elección de los valores en pugna dependen de una decisión que se escapa de los elementos jurídicos y se dirija a un carácter subjetivo del juez, pareciera ser que eso es lo que Lariguet quiere hacer entender.

Frente a la tragedia, la doctrina encuentra dos versiones: la primera es en un sentido fuerte y la segunda en un sentido débil (Pérez, 2007). Según el sentido fuerte, la tragedia consiste en que el remordimiento existirá sea cual sea la decisión, no hay base de conocimiento de los valores que diga que una decisión es más correcta que otra, porque esa preferencia sobre la decisión que se toma revela una preferencia particular o la elección de una situación particular. Mientras que el sentido débil de la inconmensurabilidad niega la existencia de la métrica común de los valores.

Hay quienes rechazan la existencia de los casos trágicos tales como Dworkin, quien supone bajo su teoría del juez Hércules, que todos los casos son fáciles, puesto que poseen un sola respuesta correcta, excluyendo la posibilidad de los casos trágicos y difíciles. Pero no se puede pretender que el Derecho sea pura lógica, porque también es experiencia, por lo que no hay razón para excluir de la experiencia de lo trágico en el Derecho (Atienza, 1997).

\section{E. ¿Un caso trágico en la jurisprudencia constitucional colombiana?}

A continuación se describe un ejemplo que se ha identificado en la jurisprudencia constitucional colombiana como caso trágico ${ }^{3}$. Se trata

3 Para más ver Mauricio Enrique RODRÍGUEZ DELGADO, Carlos Arturo DUARTE MARTíNEZ, María Andrea FIGUEROA ALFONZO, "La satisfacción de las acreencias laborales en los procesos de liquidación de las sociedades comerciales en Colombia: Historia de un caso trágico", IJ Editores, 2014, en http://www.ijeditores.com.ar/articulos.php?idarticulo=68128\&print=2, fecha de consulta: 06 de mayo de 2014. 
de la Sentencia T-458 (Corte Constitucional, 1997).

Ella versa sobre el impago de mesadas pensiónales y salarios de prestaciones sociales de pensionados y trabajadores de una sociedad, sujeta a la inspección y vigilancia de la Superintendencia de Sociedades, que fue sometida a un concordato preventivo. Los pensionados de la empresa An Son Drilling Company of Colombia, interpusieron acciones de tutela contra la empresa, al considerar vulnerados sus derechos al trabajo, a la vida, a la salud y a la seguridad social, el mínimo vital básico y de petición debido al no pago de las mesadas pensionales a cargo de la An Son, y de la suspensión del servicio médico al que tenían derecho. Los jueces y tribunales de primera instancia ordenaron el pago inmediato de las pensiones, mientras que, los jueces y magistrados de segunda instancia con algunas excepciones, revocaron las decisiones de primera instancia y denegaron el amparo constitucional solicitado.

Al final la Sala Tercera de Revisión de la Corte Constitucional compartió el razonamiento de aquellos jueces y tribunales que concedieron el amparo y ordenó a la empresa en concordato a cancelar oportunamente las mesadas pensionales, con el fin de garantizar el derecho fundamental de la seguridad social y el mínimo vital de los pensionados, que en este caso había sido violado en cuanto la empresa que se encontraba sometida al trámite de un concordato preventivo obligatorio asumió directamente el pago de la carga pensional.

En este caso la Corte hizo prelación al pago del pasivo pensional, por considerar que era el menor daño posible que podía existir con su decisión, pues consideró que por sus condiciones personales no podían acceder al mercado de trabajo y no tenían otro ingreso que aquel proveniente de su pensión, motivo por el cual se profirió la protección del mínimo vital de los lesionados.

\section{LA TRAGEDIA EN EL DERECHO}

En el apartado anterior se presentó una síntesis teórica de la argumentación jurídica, con especial referencia a la tipología de los casos, de ellos los denominados casos fáciles y difíciles que han tenido receptividad por diferentes autores. Sin embargo la controversia sobreviene en los llamados casos trágicos en dos sentidos. Primero, desde los postulados de Atienza, los casos trágicos corresponden a una clasificación en la argumentación de la decisión por el juez, pero que en sí misma no es posible. Segundo, para Lariguet, estos constituyen un 
límite a la racionalidad dada la incomensurabilidad de los valores. En ambas concepciones se observan sendas críticas al Derecho que son formuladas desde el campo iusfilosófico por cuanto dan una importancia primordial al sentido de los valores -morales- y la búsqueda de justicia.

No obstante la validez expositiva que incluye la rigurosa disertación sobre lógica formal, material y dialéctica, así como los criterios para visualizar su existencia -la del caso trágico-, la intención de este ensayo es deslindarse de este campo iusfilosófico del debate, toda vez que -al menos desde la visión que aquí se adopta- relativiza la discusión jurídica en la medida que las decisiones del juez pueden tener lecturas oponibles en las posturas filosóficas, ideológica, política, económica e incluso teológica.

Ejemplo de lo anterior es el tema del aborto, que sin duda genera posiciones encontradas entre quienes consideran vulnerado el derecho a la vida como absoluto y extensivo al no nacido, además de un agravio (pecado) a los principios religiosos, y quienes exigen la defensa de los derechos de la mujer (la dignidad, libertad, libre desarrollo de la persona individual, la salud, vida, integridad corporal, autonomía reproductiva y la igualdad con el hombre).

La Corte Constitucional colombiana en Sentencia C-355 de 2006 asumió una postura en defensa de los demandantes en tres eventos específicos. Sin ser la intensión de este escrito ahondar en el fallo, se desea resaltar que el proceso de razonamiento sistemático de la normatividad nacional e internacional, incluidos los instrumentos interpretativos de racionalidad y proporcionalidad en el estudio de los derechos, así como su examen teleológico, produjeron resultados en el plano jurídico proponiendo límites, reglamentando las excepciones a los derechos enfrentados y conceptualizando sobre los fenómenos que la componen.

Con esto se quiere decir que a pesar de la complejidad y polémica desatada, no existe, de acuerdo a los argumentos del guardián constitucional, un sacrificio o imposibilidad decisoria, pero subsiste en el plano suprajurídico el discurso de injusticia, de vulneración a la vida y los valores -morales-.

Esto lleva a considerar el tema en su amplitud e intentar dirigirlo al ámbito de la aplicación de las normas definido por Atienza (2005), de 
manera que el espacio de análisis sea extensivo al sistema normativo - de los valores constitucionales y principios del derecho- y del operador, para poder explicar que la noción de "trágico" se advierte desde: primero, el proceso mismo de creación normativa, bien por inobservancia, o bien, por contradicción de los presupuestos esenciales y materiales; segundo, la ejecución del operador que en no pocas ocasiones es mecanicista y autómata; y tercero, la decisión, donde se amplifica y evidencia dicha cadena, exhortando a una reorientación o corrección de la pugna supuesta entre derechos, valores y principios fundamentales que se asumen como inderrotables.

Para lograr este objetivo, se suman a los criterios definidos por Atienza en relación a lo razonable de la decisión jurídica, otros parámetros que responden a dos lineamientos, por una parte, el material, referido estructura jurídica y por otra parte, el sustancial, que incorpora las herramientas de interpretación y el acervo jurisprudencial -especialmente la constitucional-.

\section{1 "Otros" criterios de análisis}

\section{Materiales}

Sea legislador, juez o cualquier otro funcionario, la primera valoración exigida es determinar la legalidad de la conducta o hecho jurídico, lo cual en consecuencia condiciona la actuación del operador al sistema jurídico, pero esta situación no es óbice para rehuir al deber persistente e inacabable de observar la coherencia y armonización de la norma respecto al contexto y a los pronunciamientos de las altas cortes.

\section{Sustancial}

Abarca el desarrollo de la jurisprudencia y de los institutos jurídicos básicos por parte de las altas cortes y las decisiones que emanan de los tribunales internacionales reconocidos por el Bloque de Constitucionalidad. También a la consolidación de los instrumentos de interpretación como lo son test de racionalidad, la ponderación y el núcleo esencial.

Estos elementos, material y sustancial, permiten, por un lado, restringir la actividad en el ámbito de la aplicación de la norma y por otro, que cuando se sostienen la inaplicabilidad de una acción legal o satisfacción del derecho por inobservancia de precedente 
jurisprudencial o hasta que la autoridad inmediata superior lo permita, es decir, que se excluya alguno de estos dos elementos se generan las situaciones trágicas.

\section{HACIA UNA RESIGNIFICACIÓN DEL CASO TRÁGICO}

Según los criterios de Atienza y otros autores, se puede repensar el caso trágico no como una clasificación para la argumentación o un límite a la racionalidad, sino como una categoría de análisis crítico respecto a esos elementos que inciden en la actuación del operador jurídico en el ámbito de la ejecución de la norma.

Es así que como categoría permite hallar los nudos problematizadores en la progresión sistemática que finaliza con una decisión jurídica, es decir, podemos tomar el fallo judicial como punto de referencia para hallar las falencias, contradicciones, e inconsistencias de las normas y la actuación del operador e identificar dónde inicia el fenómeno que llevaría a la supuesta imposibilidad teórica, filosófica, ética, etc., de la decisión trágica.

Para ello hay que valerse de herramientas existentes en el Derecho como en el análisis sistemático y teleológico, pero con la salvedad de que no se buscarán los argumentos de la decisión, sino las ausencias o contradicciones entre principios, valores, conceptos constitucionales de la jurisprudencia y las normas de donde se enmarcan las acciones en litigio.

A modo de ejemplo en la T-458 (Corte Constitucional de Colombia, 1997), la Corte afirma el carácter prevalente de los pensionados frente a los trabajadores de la sociedad en mención. Si bien esta prelación no está prevista en el artículo 36 del Código Sustantivo del Trabajo (Ley50, 1990), en la que genéricamente se alude al pago de acreencias laborales, en las que caben tanto las de los pensionados como la de los trabajadores, debido a que son sujetos de especial protección constitucional, como población vulnerable, específicamente por tratarse de personas mayores de edad que están pensionadas.

De esta manera la Ley 1116 de 2006, que consagró los principios de Universalidad, igualdad, eficiencia, información y negociabilidad, hace que en adelante el operador jurídico liquidador que participe en los procesos concursales en los que se encuentre ante una situación similar, debe preferirse la población vulnerable (pensionados), por su 
condición de indefensión y la prevalencia expresa que da la Corte Constitucional que enunció tanto en la ratio decidendi como en su obiter dicta.

\section{REFLEXIONES FINALES}

En los eventos señalados por Atienza, su inconformidad versa sobre la injusticia que se provoca en el dilema del juez al tomar una de las dos opciones, lo cual lleva a preguntar si el caso trágico es una crítica al derecho o al sistema de los valores éticos y morales en un Estado.

Se crítica la postura de Atienza respecto a la imposibilidad de una decisión justa por cuanto dicho pronunciamiento es suprajurídico y por tanto la discusión se dirige a la Filosofía, la ética y la moral, generando un escenario permanente de contradicción y relatividad.

Se resignifica el "caso trágico", como parte de la clasificación exclusiva de la decisión del juez y se amplía hacia el espacio de "aplicación normativa", sustentado en lo "razonable".

El razonamiento de "trágico" dicho por la Corte se predica que cualquier decisión genera un efecto nocivo, prefiriendo la de menor costo constitucional, en ningún caso pondera valores de orden moral. Por el contrario, esta identifica que los demandantes pertenecen a una categoría que la jurisprudencia en diversos pronunciamientos ha señalado como población prioritaria para la defensa y el restablecimiento de sus derechos. De manera que al aplicar el caso trágico como categoría de análisis se pudo observar que la Ley en cuestión para resolver la controversia, no contenía en forma expresa la distinción prioritaria de la población, situación que provocó un debate jurídico para establecer la posición frente a la norma aplicada, pero en ningún momento la renuncia de un derecho frente a otro por tesis contrapuestas. Igualmente se puede alegar en adelante que las situaciones de índole similar el operador optará por el esquema definido por la Corte, así la norma no se encuentre formalmente.

\section{REFERENCIAS BIBLIOGRÁFICAS}

Atienza, M. (1987). "Para una razonable definición de «razonable»", en DOXA. Cuadernos de Filosofía del Derecho, 4, 189-200. 
Atienza, M. (2005). Las razones del Derecho. Teorías de la argumentación jurídica, México D.F., Universidad Nacional Autónoma de México.

Atienza, M. (1997). "Los límites de la interpretación constitucional. De nuevo sobre los casos trágicos", en Isonomía. Revista de Teoría y Filosofía del Derecho, 6, 7-30.

Castro, J. (s.f.) "Otra vez sobre los casos trágicos. La previsibilidad de la decisión judicial: ¿posible o utópica? tal vez necesaria. Recuperado d e http://jurastudio.blogger.de/static/antville/jurastudio/files/otra\%20ve z\%20sobre $\% 2010$ \% $\% 20$ casos $\% 20$ tragicos.pdf

Corte Constitucional de Colombia. Sentencia T458. (Septiembre 24 de 1997). M.P. Eduardo Cifuentes Muñoz.

Corte Constitucional de Colombia. Sentencia C355. (Mayo 10 de 2006). M.P. Jaime Araújo Rentería y Clara Inés Vargas Hernández.

Lariguet, G. (2005). "Conflictos trágicos y ponderación constitucional. En torno a algunas ideas de Gustavo Zagrebelsky y Riccardo Guastini”. Diritto \& Questioni Pubbliche, 5, 67-85.

Lariguet, G. "Conflictos trágicos genuinos, ponderación y límites de la racionalidad jurídica. En torno a algunas ideas de Manuel Atienza", en Isonomía: Revista de teoría y filosofía del derecho, 24, (2006), 93113.

Lariguet, G. (2004). "Conflictos Trágicos y Derecho. Posibles desafíos". Doxa. Cuadernos de Filosofía del Derecho, 27.

Ley 50 de 1990. Por la cual se introducen reformas al Código Sustantivo del Trabajo y se dictan otras disposiciones. Diciembre 28 de 1990.DO. N³9618.

Pérez, O. (2007). "Una aproximación aristotélica a la deliberación en casos trágicos", Universidad de Alcalá. Recuperado de http://www.uv.es/CEFD/15/perezdelafuente.pdf

Rodríguez, M., Duarte, C., \& Figueroa, M. (2014). “La satisfacción de 
las acreencias laborales en los procesos de liquidación de las sociedades comerciales en Colombia: Historia de un caso trágico”, IJ Editores. Recuperado de http://www.ijeditores.com.ar/ articulos.php?idarticulo $=68128 \&$ print $=2$

Vázquez, O. (2005) "La argumentación jurídica en el Tribunal Constitucional Español: los casos fáciles, difíciles...trágicos”, Madrid, Universidad Carlos III de Madrid. Recuperado de http://www.rtfd.es/ numero9/109.pdf, fecha de consulta: 04 de abril de 2014. 